\title{
MODELLING THE SPREAD OF GRASS FIRES
}

\author{
D. H. ANDERSON, E. A. CATCHPOLE, N. J. DE MESTRE and T. PARKES
}

(Received 16 February 1981)

(Revised 25 May 1981)

\begin{abstract}
A simple elliptic model is developed for the spread of a fire front through grassland. This is used to predict theoretical fire fronts, which agree closely with those obtained in practice.
\end{abstract}

\section{Introduction}

Bushfires are extremely complex and destructive natural phenomena which depend on availability of fuel, meteorological conditions and an initial ignition. The overall purpose of bushfire research is a better understanding of the phenomena so that firefighting techniques can be improved, controlled burnings can be undertaken with more confidence, and lives and property might be preserved.

One aspect of this research is to document fire behaviour during controlled burnings [6], [9] or immediately after a large fire has run wild [3], [12]. This documentation can then be used as a basis for producing and checking various models of bushfire characteristics. For example, many models have been developed to calculate forward rates of spread for various fuel complexes. A detailed list is given in Albini [1]. The most successful of these is undoubtedly Rothermel's wildland fuel spread model [10], which has been incorporated in computer-assisted approaches [2], [7] to determine the position of the fire front.

We present in this paper a simple alternative deterministic model, based on Huygens' principle, which uses an elliptical spread at each point of the fire front. We begin by considering a constant wind over a perfectly flat terrain containing a homogeneous supply of grass fuel, and progress through a single change of wind

() Copyright Australian Mathematical Society 1982 


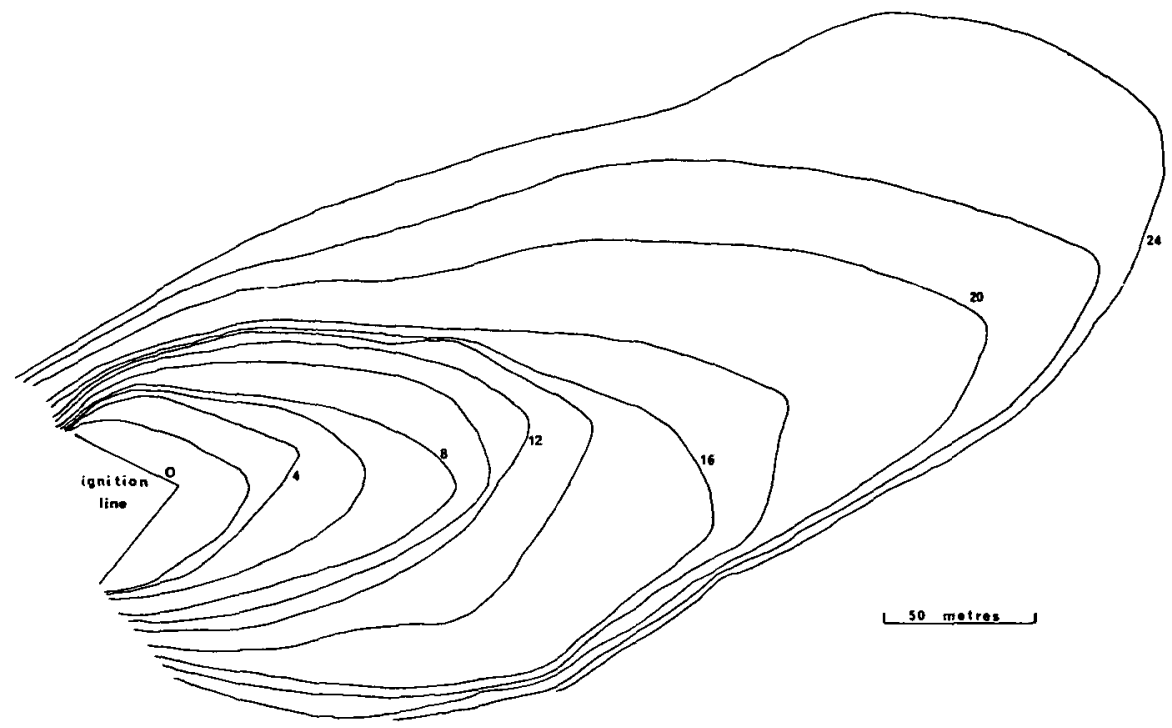

Fig. 1. Fire front contours at 2 minute intervals for experimental fire 7.

speed and direction to a variable wind. Suggestions are made also on how to handle nonuniform fuel and variable topographical conditions.

In addition our model is fitted to two experimental fires (numbers 7 and 53) from a series of controlled burns in Australia's Northern Territory carried out by N. P. Cheney of the C.S.I.R.O. Division of Forest Research between 1972 and 1976. The position of the fire front was determined by placing markers manually every 1 or 2 minutes and then using these in conjunction with field notes to sketch in the fire-front contours. The 2-minute contours for fires 7 and 53 are shown in Figures 1 and 2. Note that fire 53 was ignited at a point source whereas fire 7 was ignited along a triangular front. These fires show simple changes in direction and are well suited for a comparison of theory with experiment.

Wind speed for fire 53 was measured by a simple cup anemometer 50 metres upwind from the ignition point. For fire 7 more extensive wind data was obtained, a line of six anemometers being placed across the path of the fire, each anemometer recording the 3-dimensional wind velocity at 4 -second intervals. This yields a large amount of information which is currently being analyzed.

In both cases the anemometers were 2 metres above ground level, which was approximately half the flame height. 


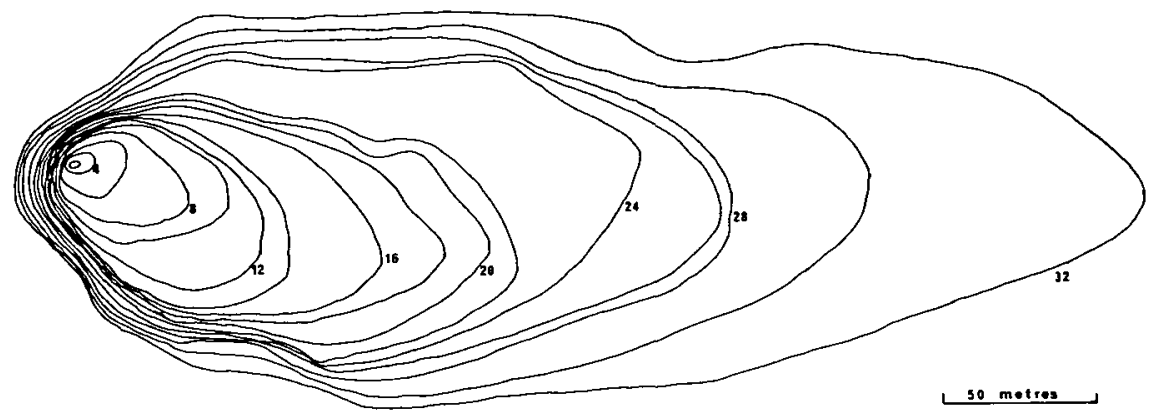

Fig. 2. Fire front contours at 2 minute intervals for experimental fire 53 .

\section{No wind}

Let us first imagine a perfectly flat terrain with a uniform cover of fuel (grass). If there is no wind blowing then conditions are isotropic, and it is reasonable to assume that under these conditions a fire started at a single point will spread out in a circular fashion. Let us further assume that the burning rate, that is the speed of advance of the fire front, is constant. This has been observed in small-scale fire experiments (Curry and Fons [4], Fons [5]) and so this assumption is reasonable, except perhaps during the early part of the life of the fire. Under this model the position of the fire front $t$ minutes after the start will be given by $x^{2}+y^{2}=a^{2} t^{2}$, or in parametric form by

$$
x=a t \cos \theta, \quad y=a t \sin \theta .
$$

The burning rate $a$ will depend on the flammability of the grass and the depth and packing density of the grass cover. The flammability of the grass is affected by factors such as the ambient temperature, humidity, and length of time since the last rainfall.

\section{Constant wind}

Suppose now that there is a uniform, constant wind blowing. We choose the $x$-axis to be in the direction of the wind. The speed of the wind is $V$, independent of $x, y$ and $t$. The wind will affect the fire by altering the angle of the flame front. This will affect, in varying degree, the speed of the head of the fire (in the positive $x$ direction), the back of the fire (in the negative $x$ direction) and the lateral speed (in the $y$ direction). The different effect of the wind on the head and back of the fire will have the effect of transporting the centre of the burnt area in the direction of the wind. 


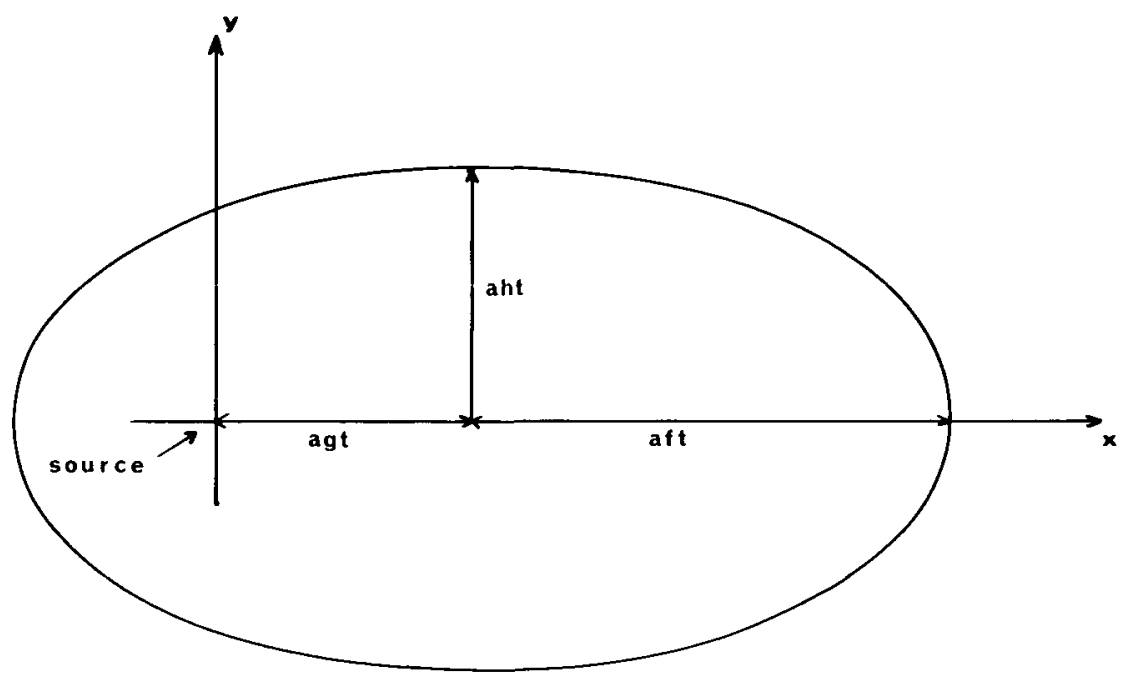

Fig. 3. Position of fire front at time $t$ under uniform wind in the $x$ direction.

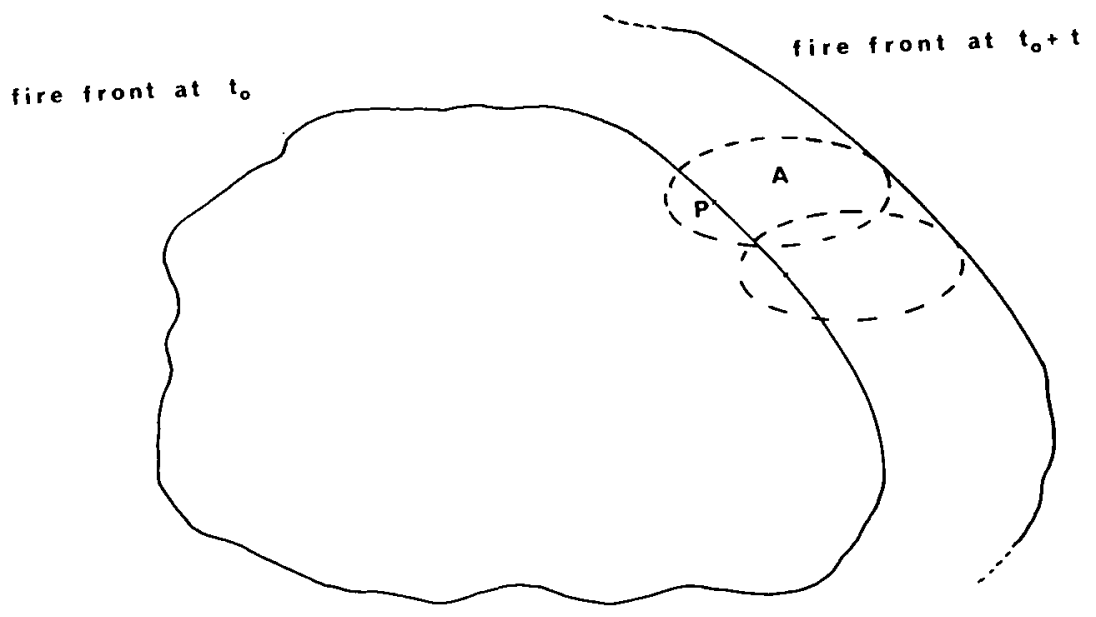

Fig. 4. Huygens' principle applied to fire fronts.

Experimental fires under roughly constant conditions appear to have an elliptical shape (see Figures 1 and 2), and like Van Wagner [11] we will take this as our model. The equation of the fire front at time $t$ is thus

$$
\left.\begin{array}{l}
x=a t(f \cos \theta+g) \\
y=a t h \sin \theta
\end{array}\right\}
$$

representing an ellipse with semi-axes atf and ath, whose centre is moving in the $x$-direction with speed $a g$, as in Figure 3. For a realistic fire front we need the 
constraints $f>g$ and $f \geqslant h>0$. This model is self-similar in time since $x, y$ and $t$ appear only in the combinations $x / t$ and $y / t$. The parameters $f, g$ and $h$ will clearly depend on the wind speed $V$, and we should write $f(V), g(V)$ and $h(V)$. The dependence of $f, g$ and $h$ on $V$ will have to be determined from experimental data. (However, to be consistent with the previous model (1) we must impose the conditions $f(0)=h(0)=1, g(0)=0$.)

A further assumption is that $f, g$ and $h$ depend only on $V$; the effects of humidity, temperature and so on being taken care of by the quantity $a$ in (2). We shall see later whether this assumption is tenable.

\section{Huygens' principle}

Having established a model for the spread of a bushfire in uniform conditions, we will now use this model together with an approach based on Huygens' principle to model the spread in non-uniform conditions.

Consider an arbitrary fire front at time $t_{0}$ (Figure 4). Imagine the fire to be suddenly extinguished and at the same time a new fire lit at one point $P$ of the fire front. This new fire is left to burn for time $t$, as in Figure 4, forming a new front. If conditions are uniform during this time from $t_{0}$ to $t_{0}+t$ then the new front will grow elliptically into the unburnt region by the previous section. Consider now the region $A$ burnt out by this new front. As the point $P$ moves around the original fire front, the region $A$ will sweep out a large region around the original fire front. Our application of Huygens' principle states that this region is in fact the region that would be burnt out by the original fire front in the time $t_{0}$ to $t_{0}+t$. Equivalently, the fire front at time $t_{0}+t$ is the envelope of the new ellipses around $P$ as the point $P$ moves along the time $t_{0}$ front. This approach is illustrated in Figure 4. Huygens' principle gives us a method of predicting the fire front at a future time if we know its present position.

\section{Consistency of Huygens' principle}

We must first of all check that Huygens' principle as described above is consistent with the model previously described. For we have now two methods for predicting the growth of a fire in uniform conditions with a steady wind: firstly, that it must grow steadily in an elliptical fashion as in Section 3; and secondly that it must grow as the envelope of small ellipses using Huygens' principle (see Figure 5). These two methods had better give the same answer. 


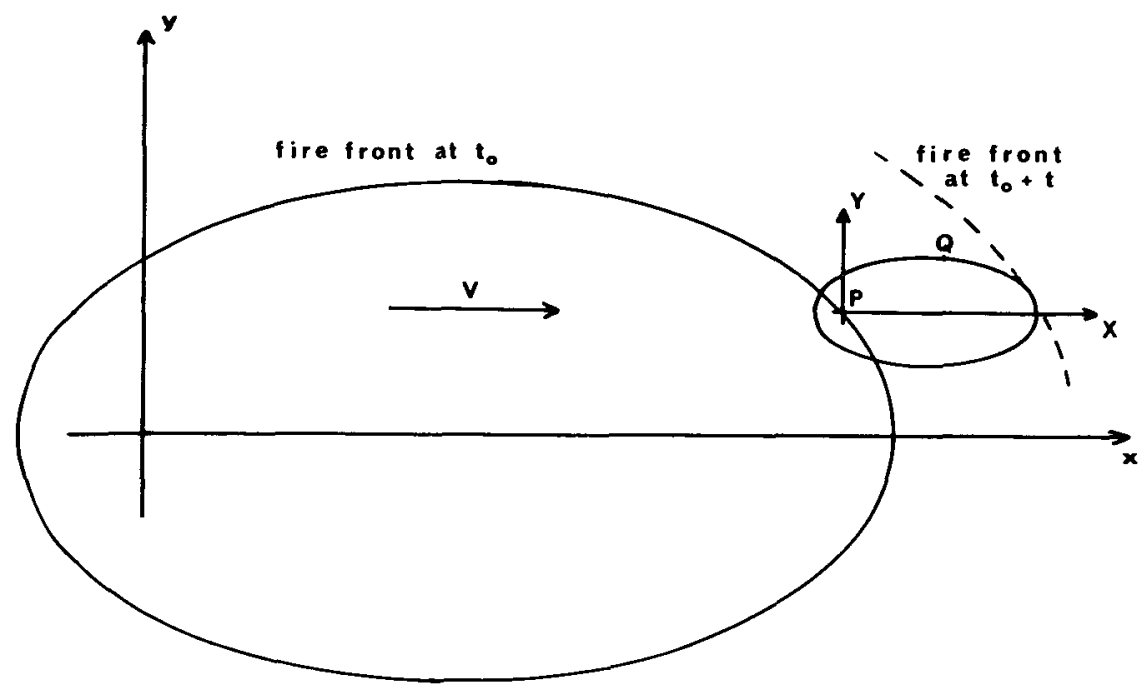

Fig. 5. Huygens' principle for a steady wind $V$.

After burning for time $t_{0}$ in a steady wind the fire front will be an ellipse, and a typical point $P$ on the front will have co-ordinates $x_{0}, y_{0}$ given by

$$
x_{0}=a t_{0}(f \cos \theta+g), \quad y_{0}=a t_{0} h \sin \theta .
$$

Let us now apply Huygens' principle to the fire front given by (3). At each point $P$ we will generate in time $t$ a hypothetical new fire front in the shape of a small ellipse. A typical point $Q$ on this small ellipse will be given by

$$
X=a t(f \cos \phi+g), \quad Y=a t h \sin \phi,
$$

where $X, Y$ are local coordinates centred at $P$ : that is,

$$
x=x_{0}+X, \quad y=y_{0}+Y
$$

are the coordinates of $Q$ with respect to the original axes (see Figure 5). From (3), (4) and (5) we can eliminate $X, Y, x_{0}, y_{0}$ and $\phi$ to obtain the equation of the small ellipse on which $Q$ lies. Letting $\theta$ vary in this equation, that is, letting the point $P$ move around the time $t_{0}$ fire front, we get a family of ellipses. The envelope of this family is, by our application of Huygens' principle, the fire front at time $t_{0}+t$. It is not difficult to show that the equation of the envelope is of the form (2) with $t$ replaced by $t_{0}+t$. In other words, in uniform conditions Huygens' principle produces the same result as the self-similar model; that is, a steadily growing ellipse moving at a constant speed downwind. We therefore proceed to use Huygen's principle to predict the behaviour of the fire front in non-uniform conditions. 


\section{A single change of wind}

As before we assume that all conditions are perfectly uniform, except that now we have a wind, still uniform in space, which varies with time. Specifically, we assume that the wind blows at a constant speed $V$ in the $x$-direction from time 0 to time $t_{0}$, and then switches through an angle $\beta$ to blow at a constant speed $W$ from time $t_{0}$ onwards. As in the previous section, we use Huygens' principle to calculate the position of the fire front at time $t_{0}+t$.

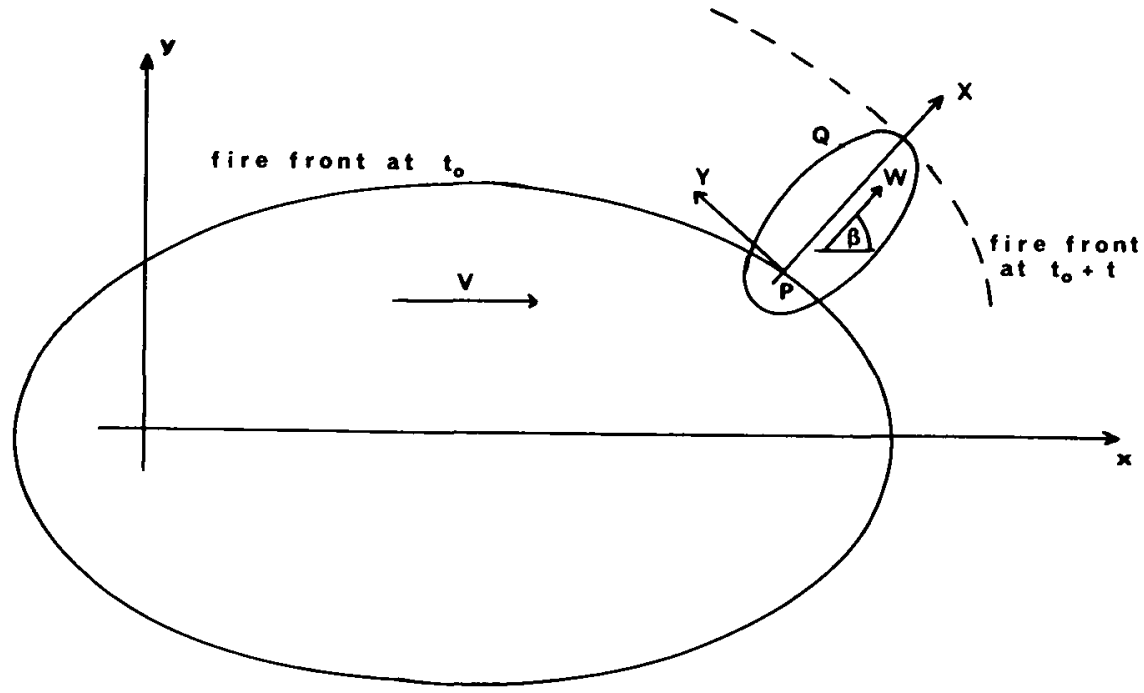

Fig. 6. Huygens' principle for a single change of wind.

Referring to Figure 6 we see that $P$ is still given by (3) and that $Q$ is still given in terms of the local axes $X, Y$ by (4), except that in (4) $f, g$ and $h$, which were understood to be $f(V), g(V)$ and $h(V)$, should now have $V$ replaced by $W$. The local axes $X, Y$ have now been rotated as well as translated, and in place of (5) we now have

$$
\left.\begin{array}{l}
x=x_{0}+X \cos \beta-Y \sin \beta \\
y=y_{0}+X \sin \beta+Y \cos \beta
\end{array}\right\}
$$

Proceeding as before we deduce from (3), (4) and (6) the equation of the envelope of all the ellipses from $P$ as $P$ moves around the time $t_{0}$ fire front. This is the equation of the fire front at time $t_{0}+t$, and for the sake of reference it is, in parametric form with parameter $\theta(0 \leqslant \theta<2 \pi)$ :

$$
\left.\begin{array}{l}
x=x_{0}+a t\left\{g(W) \cos \beta+r\left[p f^{2}(W) \cos \beta+q h^{2}(W) \sin \beta\right]\right\}, \\
y=y_{0}+a t\left\{g(W) \sin \beta+r\left[p f^{2}(W) \sin \beta-q h^{2}(W) \cos \beta\right]\right\},
\end{array}\right\}
$$


where $x_{0}, y_{0}$ are as given by (3) and

$$
\left.\begin{array}{l}
p \equiv p(\theta, \beta, V)=h(V) \cos \beta \cos \theta+f(V) \sin \beta \sin \theta, \\
q \equiv q(\theta, \beta, V)=h(V) \sin \beta \cos \theta-f(V) \cos \beta \sin \theta \\
r \equiv r(\theta, \beta, V, W)=\left[p^{2} f^{2}(W)+q^{2} h^{2}(W)\right]^{-1 / 2} .
\end{array}\right\}
$$

This new fire front is no longer elliptical, except in the special case where $\beta=0$ and the eccentricity of the small ellipse around $P$ is the same as that of the original ellipse on which $P$ lies. In practice this will occur only if there is in fact no wind change at time $t_{0}$. However as $t$ becomes large this new fire front asymptotically approaches an ellipse with major axis in the $X$ direction. For all practical purposes then, we can say that after a long time the fire "forgets" its initial shape.

As an example of the kind of fit which can be provided by the equations (7), (8) for the fire front after a single change of wind, let us look again at fire 7 (Figure 1). This fire was started on a triangular front rather than from a point. However after 16 minutes, during which the wind blew from an approximately southeasterly direction, the fire front has assumed an almost elliptical shape. We proceed to predict the spread of fire from this time on by fitting an ellipse to this 16-minute front (using nominal values $a f=7.7, a g=0, a h=3.75$ ) and then using the envelope model above to construct a predicted front for any time greater than 16 minutes.

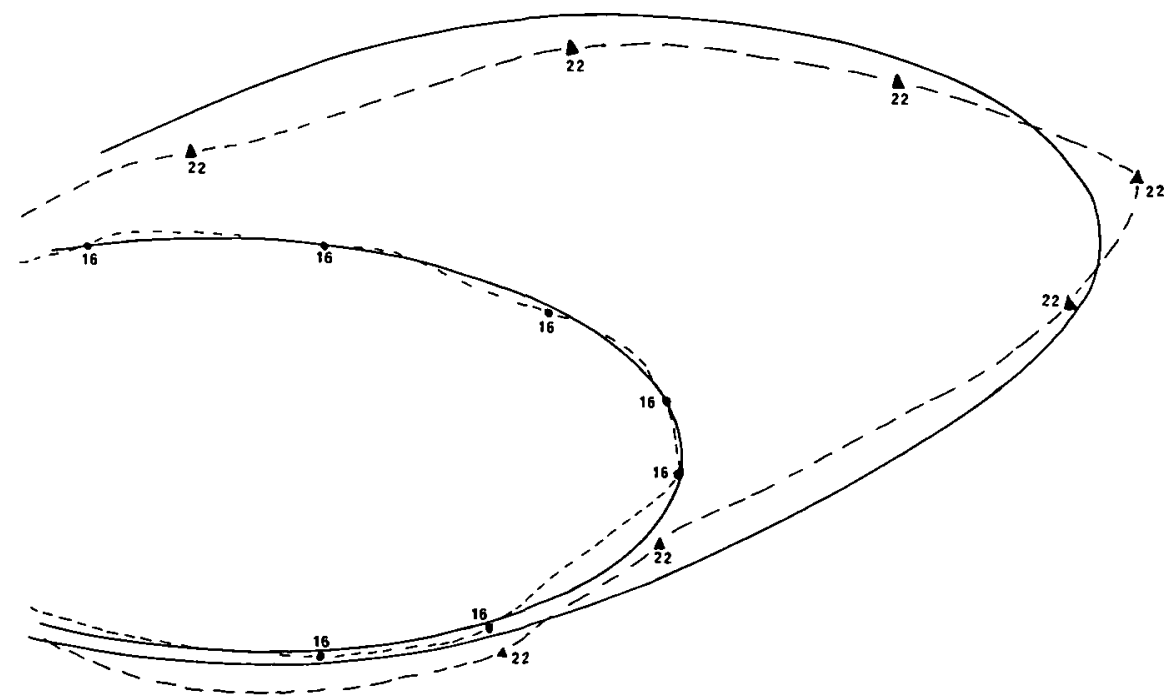

Fig. 7. Fire 7: the fitted fire front at 16 minutes and the predicted fire front at 22 minutes after a single change of wind through $30^{\circ}$ at 16 minutes. The observed fire fronts are shown dotted. 
At 16 minutes the wind appears to have veered easterly by about $30^{\circ}$ and to have remained constant for the next 6 minutes. Substituting $\beta=30^{\circ}$, and the values $a f(W)=11.75, a g(W)=10.75, a h(W)=1.75$, estimated from the observed advance of the fire front, into (7) gives the predicted fire front shown in Figure 7.

\section{Several wind changes}

As a further test of the efficacy of our modified Huygens' principle we will now fit fire contours involving several changes of wind. Unfortunately, a direct extension of the method of Section 6 is impossible because the algebra involved becomes so unwieldy that closed expressions are difficult to obtain for the front even after just two changes of wind. We describe below an approximation method, utilising Huygens' principle explicitly in incremental time steps.

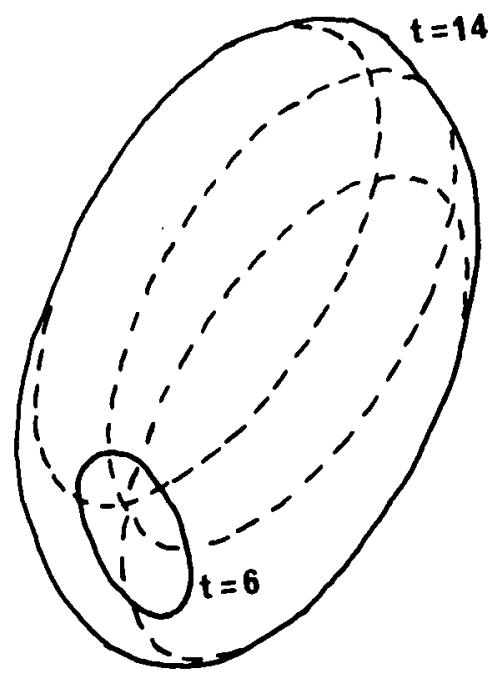

Fig. 8. Fire 53: The predicted (elliptical) fire front at 6 minutes $\left(\beta=0^{\circ}\right.$, af $=1.2$, $a g=0.8, a h=0.6$ ); the Huygens' ellipses (shown dotted) used to step from $t=6$ to $t=14\left(\beta=-55^{\circ}, a f=3.5, a g=3.1, a h=1.7\right)$; and the resulting predicted fire front at 14 minutes.

At time $t$ let the wind (still assumed uniform in space) have speed $V(t)$ in direction $\beta(t)$. From each of a large number of points $P$ on the time- $t$ fire front a small ellipse in the $\beta(t)$ direction is drawn. This ellipse, with respect to local axes $X$ and $Y$ as in the previous section, is given by

$$
X=a \delta t(f \cos \theta+g), Y=a \delta t h \sin \theta
$$




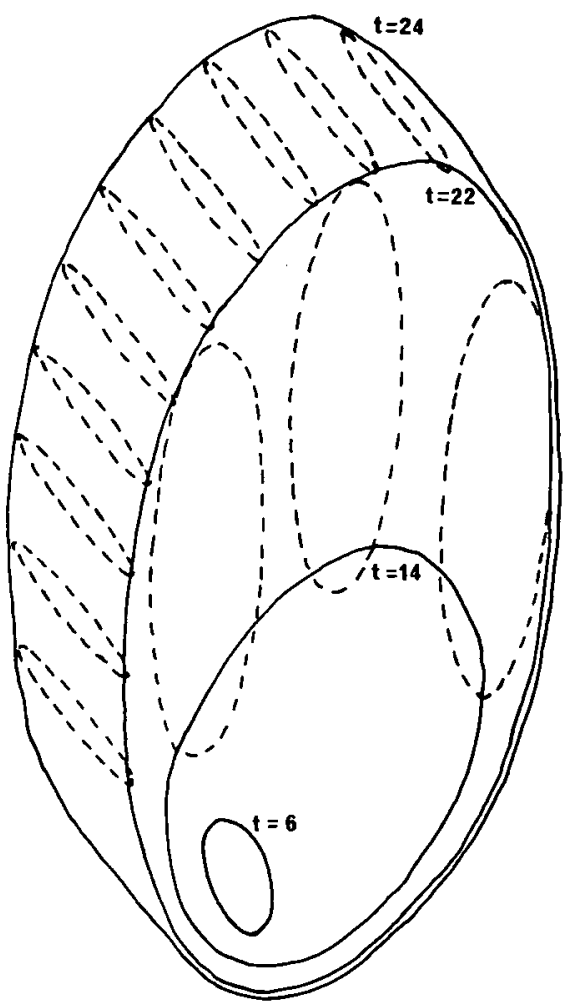

Fig. 9. Fire 53: The predicted fire fronts at $6,14,22$ and 24 minutes, and the Huygens' ellipses (dotted) used to step from $t=14$ to $t=22\left(\beta=-22^{\circ}, a f=4.2, a g=3.8\right.$, $a h=1.1)$ and from $t=22$ to $t=24\left(\beta=20^{\circ}, a f=1.9, a g=1.8, a h=0.2\right)$.

where $f, g$ and $h$ are understood to be functions of $V(t)$ and $\delta t$ is an incremental time interval small enough for the wind to be considered constant therein. The envelope of this set of ellipses is an approximation to the fire front at time $t+\delta t$. Proceeding in the same way we can predict the fire front at time $t+2 \delta t$, and so on.

We applied this method to fire 53 (Figure 2) using a graphics terminal attached to a DEC 10 computer to draw the ellipses. Looking at Figure 2 it is evident that the fire front has progressed in different directions during each of the time periods 0-6 minutes, 6-14 minutes, 14-22 minutes, and 22-24 minutes. In Figures 8 and 9 are illustrated the ellipses used to generate the approximate fire fronts using the method described above and taking $\delta t$ to be $6,8,8$ and 2 minutes respectively. In Figure 10 these approximations are compared with the observations, and the close agreement is encouraging. 


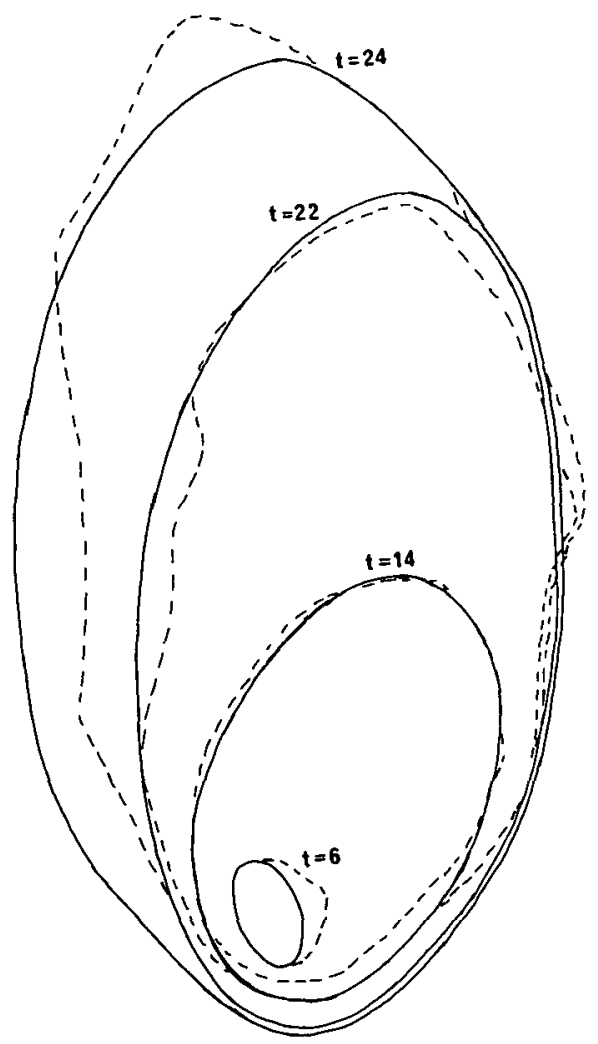

Fig. 10. Fire 53: The observed (dotted) and predicted positions of the fire front at 6, 14, 22 and 24 minutes.

\section{Non-uniform conditions}

If the wind varies spatially as well as with time, so that $V$ and $\beta$ are functions of $x$ and $y$ as well as of $t$, then the envelope method described in the previous section can still be used, although it will no longer be true that the little ellipses generated at each point $P$ (see Figure 4 ) are all identical: as $P$ moves around the fire front these ellipses will vary in size, eccentricity and orientation. Although this complicates matters somewhat, it presents no fundamental difficulties to the approach suggested in Section 7.

If it were merely a matter of modelling a spatially (as well as time) varying wind it is doubtful whether this procedure would be worthwhile. However we can use this as a device for simulating the behaviour of a fire in non-uniform conditions, that is with hilly terrain or with non-homogeneous fuel, simply by 
modifying the local wind. For example, since a fire burns rapidly up a slope, its effect on the fire can be modelled by regarding the terrain as flat and adding a virtual wind in the uphill direction. This will be dealt with in a future paper. Rothermel [10] treats wind and slope in a manner similar to this, giving the rate of spread of the head-fire as $R=a\left(1+\phi_{w}+\phi_{s}\right), \phi_{w}$ and $\phi_{s}$ being wind and slope factors respectively. If in our model we restrict attention to the wind direction only then we obtain a relationship equivalent to Rothermel's, with $\left(1+\phi_{w}+\phi_{s}\right)$ replaced by $(f+g)$.

\section{Dependence on wind speed}

The parameters involved in our model are:

$a$ : the speed of the fire front in the absence of any wind,

$f, g, h$ : when combined in the forms $f+g, h, f-g$, give the proportional changes in $a$ which occur at the head, side and back of the fire respectively, due to an external wind,

$V, \beta$ : the speed and direction of the wind.

It is certainly true that $a$ is a function of many external conditions, including ambient temperature and humidity, plus density, depth and dryness of the grass; and it is quite conceivable that $f, g$ and $h$ will depend on many of these. Although it is in no way crucial to the validity of our model, it would nevertheless be a great simplification if we could assume that $f, g$ and $h$ were in fact independent of the abovementioned quantities, and depended solely on the wind speed.

In Figure 11 are shown the forward speed of the fire front, $a(f+g)$ in our model, and the lateral speed, ah, plotted as functions of time for fire 7 , together with a plot of the wind speed against time. If the fuel is homogeneous and the terrain flat over the area of the fire, then $a$ should be constant and the graphs should represent $f+g$ and $h$ as functions of time, although their magnitudes are unknown since $a$ is not known. It is clear from Figure 11 that although the wind speed is varying only slightly there is a lot of variation in $f+g$ and $h$. There are several possible reasons for this, including inhomogeneity in the fuel, rapid fluctuations in the wind, inaccuracies in the data and the difficulty of estimating the parameters. As yet information is not available to determine which of these are important.

If $f, g$ and $h$ depend only on wind speed $V$ then the ratio

$$
\frac{\text { forward speed of fire front }}{\text { lateral speed of fire front }}=\frac{a(f+g)}{a h}
$$



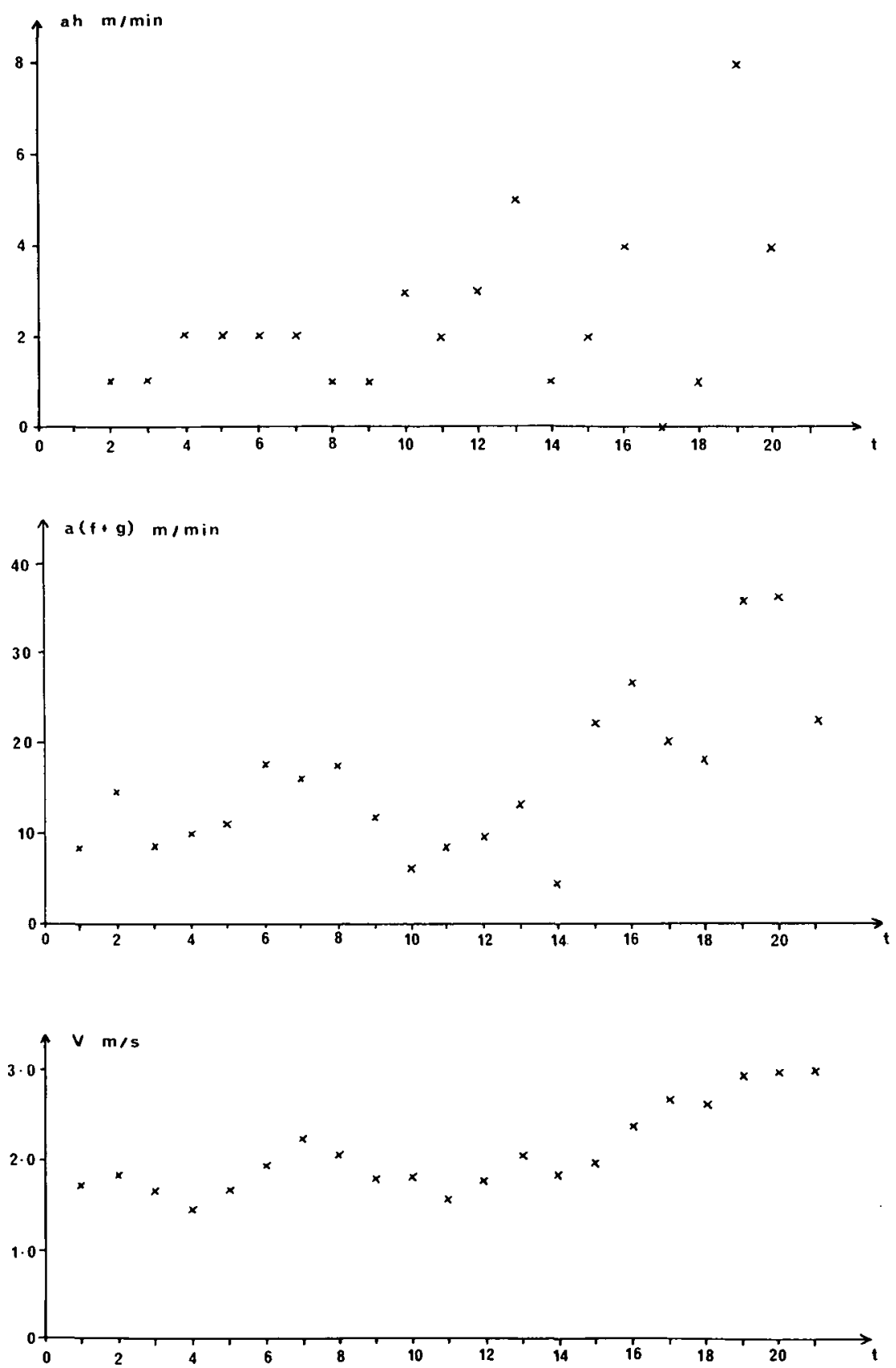

Fig. 11. Fire 7: (a) Lateral speed of fire front versus time. (b) Forward speed of fire front versus time. (c) Wind speed versus time. 
should also depend only on $V$, as is shown by Luke and McArthur ([8] Figure 6.13). Assuming homogeneity of fuel and flatness of terrain as before, we see that this ratio should be independent of ambient temperature, humidity etcetera, and so should be directly comparable from one fire to another. Mean values of the ratio $(f+g) / h$ have been calculated for various fires and are plotted in Figure 12. It appears that $(f+g) / h$ increases with growth in the mean wind speed, but the range of wind speeds for the experimental fires is not large.

All fires considered were in grassland, with tall tropical grasses dominated by Sorghum intrans, with typical stalk length of 3 metres, but lying over so that the depth of the fuel bed was 1 metre or less. The grass was fully cured, and ambient

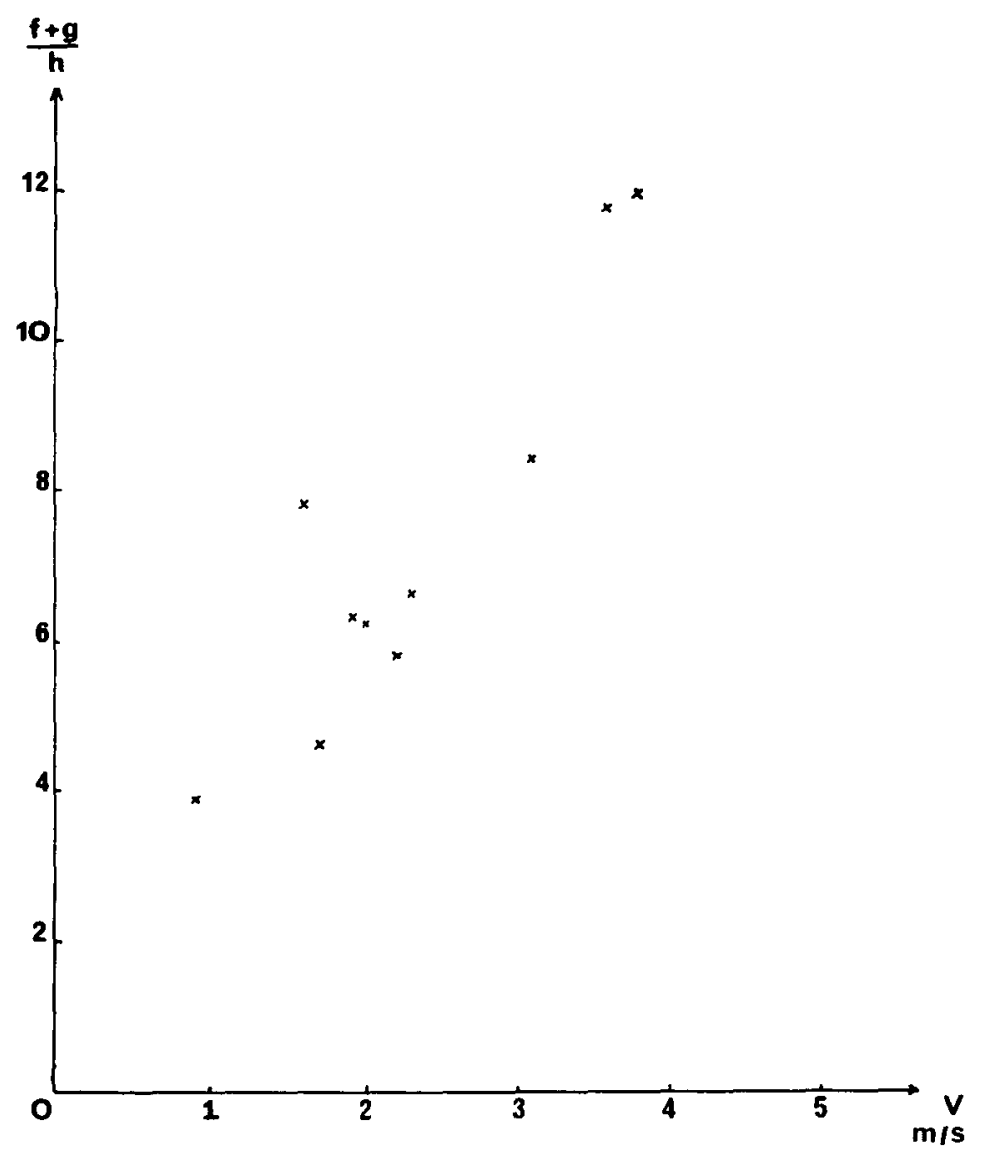

Fig. 12. Ratio of forward speed to lateral speed for various fires. 
temperature during the fires was close to $30^{\circ} \mathrm{C}$. Factors which did vary significantly among the fires were fuel load, ranging from 1.5 to 4 tonnes per hectare; relative humidity, ranging from $30 \%$ to $60 \%$; and to a lesser extent fuel moisture content, ranging from $9 \%$ to $12 \%$.

The burning rate $a$ in still wind is thus likely to have varied considerably from fire to fire. The trend shown in Figure 12 thus lends support to the hypothesis that $f, g$ and $h$ are independent of external conditions, at least for the fuel type considered, and depend only on mean wind speed.

A more extensive series of experiments is required to determine the parameters $a, f, g, h$ in the model for a wide range of wind speeds, which is necessary for its practical implementation.

\section{Conclusion}

We have proposed a deterministic model for the spread of fire fronts under idealized conditions, and found that this model is likely to be flexible enough to handle real situations with non-constant wind, varying fuel, and non-flat terrain. We have tested the model by using it to fit the observed fire fronts from two of a series of grassland fires conducted by the CSIRO Division of Forest Research. The two fires chosen are fairly typical. The fit achieved was good enough to be encouraging.

Data is available for the wind speed $V$, and in some cases for the 3-dimensional wind velocity, as well as for the position of the fire fronts, but in this paper we have not used this wind information in fitting the model fire fronts to the observed data. We have merely investigated whether suitable values of the parameters could be chosen to provide a good fit to the data; in other words, whether our elliptical model was viable.

Present research is being directed into the automatic prediction of the position of the fire front using this elliptical model as a foundation. To do this we will need to know the value of the parameter $a$ for various fuels and weather conditions, and be able to express the parameters $f, g$ and $h$ as functions of $V$. An attempt was made using the available data to determine the dependence of $f, g$ and $h$ on $V$, but only the dependence of $(f+g) / h$ for a limited range of $V$ could be found. Testing the predictive value of the model will involve trying to fit the fire not by an ad hoc choice of $f, g$ and $h$ but by actual measurement of wind speed $V$, and then using the appropriate values of $f(V), g(V)$ and $h(V)$ with an estimate of $a$. In order to do this further experiments will need to be carried out. 


\section{Acknowledgements}

We are grateful to Phil Cheney of the CSIRO Division of Forest Research for access to his experimental data, and for helpful discussions on the nature of bushfires. We would also like to thank the referees for their valuable comments.

\section{References}

[1] Frank A. Albini, "Estimating wildfire behaviour and effects", USDA For. Serv. Gen. Tech. Rep., INT-30 (1976).

[2] Frank A. Albini, "Computer-based models of wildland fire behaviour: a users manual", Intermt. For. and Range Exp. Stn., Ogden, Utah, (1976).

[3] Hal E. Anderson, "Sundance fire: an analysis of fire phenomena", USDA For. Serv. Res. Pap., INT-56 (1968).

[4] J. R. Curry and W. L. Fons, "Rate of spread of surface fires in the Ponderosa pine type of California", J. Agric. Res. 57 (1938), 239-267.

[5] Wallace L. Fons, "Analysis of fire spread in light forest fuels", J. Agric. Res. 72 (1946), 93-121.

[6] Richard W. Klukas, "Control burn activities in Everglades National Park", Proc. Annual Tall Timbers Fire Ecologv Conf., Tall Timbers Res. Stn., Tallahassee, Florida (1973).

[7] P. H. Kourtz and W. G. O'Regan, "A model for a small forest fire... to simulate burned and burning areas for use in a detection model", For. Sci. 17 (1971), 163-169.

[8] R. H. Luke and A. G. McArthur, "Bushfires in Australia", Aust. Govt. Publishing Service, Canberra (1978).

[9] G. B. Peet, "The shape of mild fires in jarrah forest", A ust. For. 31 (1967), 121-127.

[10] Richard C. Rothermel, "A mathematical model for predicting fire spread in wildland fuels", USDA For. Serv. Res. Pap. INT-115, (1972).

[11] C. E. Van Wagner, "A simple fire-growth model", For. Chron. 45 (1969), 103-104.

[12] Dale D. Wade and Darold E. Ward, "An analysis of the Air Force Bomb Range Fire", USDA For. Serv. Res. Pap., SE-105, (1973).

Department of Mathematics

Faculty of Military Studies

Royal Military College

Duntroon

A.C.T. 2600 